recibido: 16.01 .13 / aceptado: 11.04 .13

\title{
Retos de la radio en los escenarios de la convergencia digital
}

\author{
Xosé Soengas Pérez \\ Universidad de Santiago de Compostela
}

\section{Palabras clave}

Convergencia digital, radio, multimedia, contenidos radiofónicos, programación radiofónica, internet

\section{Resumen en castellano}

Este artículo analiza la situación actual de la radio española en el nuevo escenario mediático, caracterizado por la convergencia digital, la redefinición de las funciones clásicas de los medios y la evolución constante de una audiencia cada vez más segmentada y más exigente. En la nueva era de la información cambian completamente los códigos y las normas vigentes durante décadas, especialmente aquellas que afectan a los procesos de producción y acceso a la información. Gracias a Internet los contenidos de cualquier soporte están al alcance de los ciudadanos en cualquier momento y desde cualquier lugar, y la radio ya no es el único medio con el don de la instantaneidad y de la ubicuidad. Al perder la exclusividad de una de sus principales características diferenciales tiene que adaptarse a la nueva realidad y competir también con la prensa y con la televisión, que a través de sus páginas web ofrecen servicios informativos y de ficción en condiciones similares a la radio. 


\title{
Radio challenges in digital convergence sceneries
}

\section{Keywords}

Digital convergence, radio, multimedia, radio contents, radio programming, Internet

\begin{abstract}
This article analyzes the current situation of the Spanish radio in the new media scenery, characterized by digital convergence, the redefinition of the classical functions in the media and the constant development of an increasingly more demanding and segmented audience. In the era of information, the codes and rules existing during decades have changed completely, especially those which affect the production process and the access to information. Thanks to the Internet, contents of any kind are at reach for citizens at any place and any time, and radio is no longer the only one with the gift of instantaneity and ubiquity. As it loses the exclusivity of one of its most defining characteristics, radio has to adapt itself to the new reality and compete with the press and television, which offer news and fiction services in similar conditions to radio.
\end{abstract}

\section{Autor}

Xosé Soengas [jose.soengas@usc.es] es catedrático de Comunicación Audiovisual en la Universidad de Santiago de Compostela. Sus investigaciones están centradas en el ámbito de la información y de la ficción audiovisual, especialmente en el análisis de los contenidos informativos de radio y de televisión.

Entre sus publicaciones destacan: Informativos radiofónicos (2003); El tratamiento informativo del lenguaje audiovisual (2003); Los contenidos informativos en las televisiones generalistas (2005); La información en televisión: espectáculo y realidad manipulada (2007); El control de la información en televisión (2007); El enfoque informativo. Los puntos de vista de una noticia en televisión (2008); Principales modelos de televisión en Europa (2009); Los límites de la información en los debates pactados (2009); Ficción radiofónica (2010); La realidad informativa en EE.UU., Europa y Latinoamérica (2012) y El nuevo escenario informativo en España después de la implantación de la Televisión Digital Terrestre (2013). 


\section{Introducción}

La revolución tecnológica transformó hasta tal punto el mundo de la comunicación que obligó a reestructurar los medios y a redefinir sus funciones para adaptarse a la nueva situación porque en la era digital la mayoría de las cabeceras incorporaron servicios que antes eran exclusivos de otros soportes. Así el escenario de la convergencia mediática se caracteriza por una renovación integral donde prensa, radio y televisión comparten servicios que antes correspondían únicamente a uno de ellos (Campos Freire, 2011). Y en este proceso uno de los medios más afectados ha sido la radio porque, gracias a Internet, tanto la prensa como la televisión se han apropiado de la ubicuidad y de la instantaneidad, dos características que hasta hace poco eran exclusivas de la radio.

En primer lugar, es importante situar la convergencia digital en un contexto marcado y condicionado profundamente por la tecnología, algo que ha repercutido en el descuido de la calidad de los contenidos. Pero la convergencia digital no se puede analizar sin tener en cuenta las reestructuraciones empresariales y la crisis de los medios de comunicación (Rojo Villada, 2006), unos factores que han determinado el diseño y la configuración del actual mapa radiofónico.

La evolución de los medios de comunicación no se ha producido siempre de una forma lógica, ni acorde con los cambios naturales que continuamente experimenta la sociedad, unas veces por impedimentos económicos y otras por falta de iniciativa de los empresarios, en el caso de los medios privados, y de los políticos, en el caso de los medios públicos (Reig, 2011). Por eso, para conocer bien el escenario actual hay que analizar una serie de factores que han marcado el proceso e influido en la configuración de la situación actual (Campos Freire, 2010). Como ya se ha dicho, los medios de comunicación en varias etapas de su historia han apostado por la renovación tecnológica frente a la calidad de los contenidos y han descuidado la actualización de la oferta, de acuerdo con la evolución y con las nuevas necesidades de la sociedad. Y tampoco han modernizado los formatos, algo que se ha traducido en un desequilibrio importante y en una infrautilización de los recursos materiales de la radio, debido a la falta de correspondencia entre una sofisticada infraestructura tecnológica y una programación obsoleta y de baja calidad. Pocas emisoras han diseñado desde el primer momento de la digitalización una oferta adecuada estrictamente a las necesidades actuales de una audiencia marcada, cada vez más, por la segmentación, por la fragmentación y por la especialización. La mayoría han corregido los errores o renovado los contenidos puntualmente según los resultados de audiencia, pero no por iniciativa propia.

La evolución de la radio hay que enmarcarla en la evolución de la sociedad, que se ha acentuado en las dos últimas décadas, debido, sobre todo, a la globalización, un fenómeno que ha forzado y acelerado profundos cambios en las estructuras de los medios de comunicación, algo que ha contribuido de una manera 
decisiva a la convergencia mediática. Y aquí ha jugado un papel determinante Internet.

En la nueva era de la información han cambiado completamente los códigos y las normas vigentes durante décadas, muchas de las cuales ahora quedan totalmente inservibles, especialmente aquellas que afectan a los procesos de producción y de acceso a la información, que ya se rigen por otros parámetros. Y precisamente aquí es donde se advierte uno de los principales problemas de la radio actual: la convivencia en algunas emisoras de infraestructuras tecnológicas modernas con organigramas tradicionales salpicados de formatos poco novedosos, porque la renovación no siempre se ha hecho de forma integral, lo cual genera una serie de choques o desajustes que impiden optimizar las posibilidades que ofrece la tecnología y dar respuesta a las necesidades de la audiencia.

\section{Objetivos, hipótesis y metodología}

El objetivo de este artículo es analizar la situación actual de la radio en España y conocer las fortalezas y las debilidades de este medio en un contexto marcado por la convergencia mediática, por la crisis económica y por una audiencia en constante evolución.

Partimos de la hipótesis de que la radio todavía no ha adaptado completamente su modelo a las necesidades de la sociedad actual, ni ha planteado una oferta capaz de hacer frente a la competencia que llega desde otros soportes, gracias a los nuevos modos de consumo informativo y de ficción que posibilita Internet.

Para llevar a cado este análisis hemos tomado como referencia la programación radiofónica de la segunda semana de octubre de 2012, observando las características principales de los contenidos que integran la parrilla. Nos hemos fijado, sobre todo, en las duplicidades que existen en la oferta y en la estructura de los formatos, dos elementos que consideramos esenciales para realizar una valoración cualitativa de la oferta radiofónica actual.

\section{Análisis de los datos}

Para conocer los pormenores de la radio en el escenario mediático del siglo XXI, analizamos la situación centrándonos en los principales aspectos que configuran las circunstancias que favorecieron y posibilitaron la convergencia digital: un contexto marcado por la globalización y por los cambios derivados de ella, la incorporación de la tecnología a todas las fases del proceso de producción, la obligada redefinición de las funciones tradicionales de los medios, la evolución de la audiencia y la situación económica actual, que ha afectado directamente a la estructura y a la viabilidad de los medios de comunicación (Peñafiel, 2007). 
Nos situamos en los escenarios que han intervenido en el desarrollo de la convergencia para señalar, describir y analizar con precisión los principales retos a los que se enfrenta la radio en el nuevo contexto digital (Martínez Costa, 1997). Para ello es necesario conocer las características y las necesidades de cada entorno.

\section{Los retos derivados de un contexto multimedia y de una sociedad digital en continuo cambio debido a la globalización}

Lo primero que se observa al analizar las características principales de la programación radiofónica que existe en España en estos momentos es una importante duplicidad de contenidos y una gran similitud entre los formatos de las diferentes cadenas. Si comparamos la oferta actual con una parrilla de hace 10 años, la dimensión de los cambios no se corresponde con el grado de evolución de la sociedad desde entonces. La mayoría de los espacios apenas han cambiado de estética ni de estructura, a pesar de las posibilidades que brinda la tecnología. Este es un ejemplo del alto nivel de desaprovechamiento que existe de los recursos radiofónicos desde el punto de vista estructural.

La radio es un medio integrado en la sociedad y, por lo tanto, también refleja todas las demandas que existen en ella porque la realidad es la que impone la selección de los contenidos. Desde su aparición ha experimentado varias transformaciones, pero en las dos últimas décadas los cambios han sido drásticos, debido al fenómeno de la globalización, impulsado por la tecnología (Terceiro, 1996).

El gran reto de la radio en el escenario de convergencia digital es integrar en su oferta las nuevas necesidades que se derivan de un contexto totalmente diferente al de hace dos décadas, porque la programación actual todavía no responde en su totalidad a estas exigencias, especialmente en el caso de las emisoras más pequeñas. En primer lugar, la radio no puede obviar los cambios estructurales y sociales que han acompañado la llegada del siglo XXI y que han modificado sustancialmente la relación tradicional que existía entre los medios y los ciudadanos (Saiz Olmo, 1998). Ahora en la sociedad hay otras exigencias informativas y comunicativas y, lo más importante, nuevas formas de consumo mediático y nuevos escenarios para exhibir los productos audiovisuales, que obligan a los medios a resituarse y a posicionarse de acuerdo con los nuevos parámetros en los que se ubican los distintos sectores de la audiencia. Los cambios sociales y el proceso de renovación profunda en los medios de comunicación no se pueden desligar del impacto de Internet, que requiere una renovación para hacer viables unas estructuras empresariales y unas ofertas de programación ya obsoletas. Y aquí es donde aparecen los principales desafíos de la radio ante los retos de la convergencia digital (Martínez Costa, 2001). Adaptarse a las nuevas circunstancias implica una redefinición importante de los esquemas que regían hasta ahora las directrices de la programación. Como ya se ha dicho, las ven- 
tajas tradicionales que tenía la radio sobre los demás medios de comunicación desaparecen en el nuevo contexto. Gracias a Internet, tanto la prensa como la televisión pueden competir con las ondas en rapidez y en ubicuidad y, además, simplificar y abaratar los costes que suponían los procesos de producción de la información, que eran dos obstáculos importantes (Corredoira, 1999). Por eso la radio clásica ahora se encuentra con una competencia inesperada y difícil de sortear. A esto hay que añadir la proliferación repentina de numerosas emisoras a través de Internet que ofrecen alternativas de programación atractivas, innovadoras y desprendidas de los corsés que todavía tienen algunas emisoras tradicionales, unas ataduras de las que, a veces, resulta difícil desprenderse, debido a las imposiciones que se derivan de unas estructuras muy consolidadas.

El escenario digital (Campos Freire, 2011) sitúa a la radio en un contexto muy competitivo y muy exigente desde el punto de vista de los contenidos, a lo que hay que añadir los demás desafíos estructurales propios de la convergencia mediática. Por eso los principales cambios deben orientarse con la intención de definir y establecer un modelo radiofónico viable desde el punto de vista económico, útil desde el punto de vista informativo y estructurado conforme a las nuevas características y necesidades de la audiencia.

También hay que tener en cuenta que, debido a la globalización, la radio actual ya no puede tomar como referencia exclusiva sólo lo que existe en su entorno, porque ahora, gracias a Internet, los ciudadanos tienen acceso universal a los contenidos y la competencia puede llegar de fuera.

\section{La tecnología y los cambios en la radio}

La tecnología siempre ha sido el gran aliado de la radio, y lo sigue siendo, pero, curiosamente, en las dos últimas décadas también le ha traído una gran competencia ya que, debido a los avances, otros soportes han incorporado servicios que tradicionalmente eran exclusivos de la radio y, además, marcaban una diferencia cualitativa. Algunas de las características que diferenciaban la radio de los demás medios de comunicación eran la ubicuidad, la instantaneidad y la movilidad, y estas singularidades influían directamente en su éxito entre la audiencia porque ofrecía unas condiciones de escucha con las que no podían competir ni la prensa ni la televisión. Pero estas particularidades dejaron de ser exclusivas de la radio con la llegada de Internet, que introdujo la posibilidad de una comunicación globalizada, instantánea y accesible desde cualquier lugar y en cualquier momento, independientemente del soporte (Corredoira, 1999). Y en estos momentos prácticamente todos los periódicos y todas las televisiones disponen de páginas web a través de las que ofrecen contenidos en condiciones de acceso similares a la radio: actualización permanente de la información y posibilidad de consulta universal. Así, de repente, la radio se encuentra en un escenario donde tiene que competir con otros soportes, algo impensable hace pocos años. 
Pero las renovaciones tecnológicas no siempre se han hecho con criterios profesionales, sino que muchas veces los cambios obedecían a decisiones y estrategias empresariales, pensadas desde una óptica multimedia, donde la radio estaba en desventaja con respecto a la prensa y a la televisión, que siempre han tenido un trato preferente por parte de los empresarios de la comunicación (Martínez Soler, 1998).

La radio no es ajena a la renovación tecnológica (Merayo, 1996). A lo largo de su historia ha asimilado con naturalidad varios procesos de cambio, como la informatización de las redacciones a finales de los años 80 y principios de los 90 (Saiz Olmo, 1995). Pero la llegada de Internet supuso una transformación integral y afectó a todo el sistema, modificando o dejando inservibles muchas de las funciones del organigrama clásico (De Aguinaga, 2002), especialmente todo lo referente al proceso de producción de la información. El nuevo escenario, marcado por Internet y por la digitalización (López Vidales, 2005), dejó a la radio sin la exclusividad de algunas de sus principales armas, que pasaron a formar parte de la oferta multimedia de los demás soportes. Por otra parte, el paso de la radio analógica a la digital se gestionó, en la mayoría de los casos, desde una óptica estrictamente tecnológica (López Vidales, 2000), olvidando que la nueva situación requería también una actualización de los contenidos.

Los cambios tecnológicos llevan implícitos cambios estructurales que afectan al funcionamiento de las emisoras desde diversas ópticas: acceso más rápido a las fuentes, opciones de incorporar nuevos formatos, mayores posibilidades de participación de la audiencia en espacios radiofónicos, y agilidad y simplificación en los procesos de producción de la información. Pero estas ventajas implican también una mayor competencia porque al aumentar las posibilidades aumenta la oferta (Ortiz y López, 2011). Además, la tecnología también conlleva una gran dependencia y un alto coste si se incorporan todas las novedades que, además, en la mayoría de los casos, exigen una renovación periódica y a corto plazo. En este sentido, de nuevo, se produce una brecha, porque no todas las emisoras tenían la misma solvencia y, por lo tanto, algunas estaban en situación de poder asumir los cambios propios de la modernización y de la convergencia digital y otras no. Y aquí es donde hay que situar otro de los retos importantes. Buena parte del éxito de las emisoras de radio, en un futuro inmediato, va a depender de su capacidad para adecuar sus estructuras de forma coherente a una oferta viable y novedosa, que suponga una alternativa a lo que ya existe en el mercado y que sea capaz de atraer la atención y cautivar a un sector concreto de la audiencia y consolidar esa posición. Y en este proceso juega un papel determinante la tecnología, un elemento que no se puede desligar del proceso de producción y de la calidad de los contenidos. Porque lo que se trata es de optimizar las infraestructuras y aprovechar todos los recursos para ofrecer productos competitivos. Se trata de buscar y de conseguir una alianza perfecta, o por lo menos operativa, entre la tecnología y la creatividad para hacer frente y 
dar respuesta a los retos que plantea la radio actual, que serán los que sienten las bases de la radio del futuro (Martínez Costa, 1997).

Los recursos técnicos ofrecen las posibilidades para plantear una oferta competitiva, pero para que esto sea realidad es necesario un aporte de imaginación, y ésta es la tarea más complicada y corresponde a los recursos humanos de cada emisora.

La tecnología tiene dos dimensiones que deben ser explotadas de forma óptima y complementaria: la funcional y la estética. La dimensión funcional facilita las condiciones necesarias para la emisión e influye en todos los procesos que afectan al acceso y al tratamiento de la información, y también a la innovación de los formatos, que posibilitan nuevas formas de narrar y de transmitir. Y la dimensión estética dispone de múltiples recursos que, combinados adecuadamente con los elementos anteriores, sirven para reforzar la calidad y la credibilidad de los contenidos.

La tecnología también ha diversificado y multiplicado las posibilidades de emisión y de escucha de la radio a través de otros soportes como los ordenadores y los teléfonos móviles (Cebrián Herreros, 2001). Y aquí de nuevo aparece el reto de la competencia con la prensa y con la televisión porque Internet también les ofrece las mismas opciones. Pero esta diversidad de soportes a través de los que se puede escuchar la radio permite ofrecer contenidos específicos para audiencias muy concretas, que es la tendencia actual. Esta puede ser una buena oportunidad para la radio: aprovechar los distintos espacios que se presentan en el nuevo entorno multimedia para ofrecer productos alternativos y conseguir llegar a sectores de la audiencia que no tienen las necesidades informativas o de ficción cubiertas por la prensa o por la televisión. Se trata de explotar de forma estratégica las nuevas formas de difusión que son posibles gracias a la tecnología y convertirlas en alternativas que complementen las emisiones tradicionales de la etapa analógica, o que cubran las carencias y las nuevas demandas que se presentan para la radio en la era de la convergencia digital (Prado y Franquet, 1998).

\section{La redefinición de las funciones tradicionales de los medios y los nuevos modos de recepción}

Los medios de comunicación durante décadas han tenido una serie de funciones propias que configuraban su identidad. Así cada soporte respondía a unas características específicas que limitaban y definían sus atribuciones de forma clara y concreta. Pero esto se termina con la llegada de Internet, que posibilita una sociedad multimedia. Y este fenómeno afecta tanto a la estructura de los medios, que cambian el enfoque de sus actividades, como a los contenidos, sobre todo a la información y a los espacios de actualidad. Durante este proceso de transformación la mayoría de las empresas de comunicación se embarcaron en una aventura empresarial multimedia (Martínez Soler, 1998), especialmente las 
cabeceras de prensa, derivando parte de sus inversiones hacia el sector audiovisual, y prácticamente todas pasaron a tener una o varias emisoras de radio y de televisión. Esta nueva situación cambia completamente el escenario de la comunicación y obliga a redefinir las funciones de los medios porque la mayoría de los soportes incorporan características y servicios que antes eran exclusivos de otros. Ahora podemos escuchar la radio por Internet mientras vemos imágenes y textos que ilustran las noticias que nos cuentan los locutores, las páginas webs de la prensa ofrecen entrevistas e informaciones con sonidos e imágenes en movimiento y la televisión se puede ver en el ordenador desde cualquier lugar. Además, tanto la prensa como la televisión actualizan sus contenidos periódicamente de la misma forma que lo hacía antes la radio en los boletines horarios. Por lo tanto existe un solapamiento o confluencia de funciones y se puede acceder a servicios prácticamente idénticos a través de la plataforma de cualquier soporte. Y esta novedad, fruto de las posibilidades que ofrece la tecnología, sitúa a la radio en un contexto que le obliga a buscar estrategias para conseguir audiencia, porque ahora el soporte ya no es un elemento diferenciador de la prensa o de la televisión.

Pero una de las mayores novedades en la nueva radio es la permanencia de los contenidos y la posibilidad de acceder a ellos y consultarlos en cualquier momento (Corredoira, 1999), algo que antes estaba reservado exclusivamente a la prensa. La superación de la no retornabilidad puede favorecer, sobre todo, la recuperación de la ficción como una propuesta de programación porque ahora es posible el seguimiento de relatos de forma discontinua y sin el condicionante de la rigidez horaria.

Las transformaciones en la radio no afectan sólo al proceso de producción de los contenidos. La evolución tecnológica posibilita unas formas y unos modos de recepción que modifican completamente las variables que deben tener en cuenta las emisoras para satisfacer las necesidades de la audiencia. Además, los nuevos modos de recepción también han generado nuevos consumidores, con nuevos gustos y nuevas exigencias, a los que la radio no puede ser ajena si quiere mantener el protagonismo que le corresponde en el escenario multimediático que caracteriza el panorama actual (Campos Freire, 2011).

\section{La evolución de la audiencia}

Durante décadas la radio ha tenido una audiencia consolidada que, al margen de las oscilaciones naturales, se consideraba propia de este soporte. Era una audiencia fiel y asidua que prefería la radio frente a los demás medios. Pero los numerosos, drásticos y repentinos cambios que se han producido en el panorama mediático han desestabilizado esa fidelidad, debido, entre otras razones, a una oferta más diversificada y a la evolución de los gustos y de las necesidades informativas y comunicativas de los ciudadanos. El perfil del oyente del siglo XXI es muy distinto al de hace sólo dos décadas. Y en estos cambios tiene mucho 
que ver el impacto de la globalización, que ha fomentado la apertura a otras realidades y ha modificado las costumbres e incluso las preferencias de los oyentes. Por lo tanto, la evolución de la audiencia es, posiblemente, el factor más importante a la hora de señalar los retos de la radio en el escenario actual. Así, el éxito de la radio dependerá de su capacidad para hacer frente a las nuevas necesidades y a las exigencias de una sociedad en constante proceso de cambio. Y esta evolución hay que analizarla desde dos puntos de vista: por una parte, los cambios naturales que se producen en todos los sectores de la población desde el punto de vista sociológico y, por otra parte, el acceso de los ciudadanos a todo tipo de contenidos, gracias a Internet y a la globalización. Este último aspecto ha generado unos cambios sustanciales, motivados por la influencia de ofertas desconocidas hasta el momento y también por la posibilidad de acceder a productos muy novedosos que han cambiado las preferencias de muchos sectores.

La evolución de la sociedad exige adaptar no sólo las ofertas de contenidos, sino también las estructuras de algunas emisoras, ancladas todavía en unos modos tradicionales de producción que resultan incompatibles con los ritmos y con las propuestas actuales (Ortiz y Cuesta, 2003).

Las características de la audiencia se convierten en un factor determinante que las emisoras deben tomar como referencia principal a la hora de diseñar y de programar sus contenidos. Ahora la competencia es numerosa y ya no existe la audiencia cautiva de antes, obligada a sintonizar un determinado tipo de programas porque no existían alternativas.

Los cambios en los modos de recepción y las nuevas posibilidades informativas y comunicativas que existen en el panorama mediático sitúan a la radio en unas condiciones de competencia permanente con la prensa, con la televisión y con Internet (León, 2000). Por lo tanto, lo solución consiste en plantear una oferta lo más especializada posible, capaz de destacar y de diferenciarse de las demás opciones porque la competencia de la radio ya no está sólo entre las diferentes emisoras, también existe con los demás soportes que ofrecen contenidos de características similares y en las mismas condiciones.

La solución es una oferta diversificada, adecuada a las necesidades de una audiencia fragmentada, especializada y exigente. Las emisoras no pueden mantener estructuras de programación y fórmulas idénticas. Deben especializarse de acuerdo con la demanda. Pero no basta con propuestas centradas en ámbitos genéricos, como las que ya ofrecen las emisoras temáticas, sino que se requieren alternativas más personalizadas dentro de la propia tematización.

\section{Adecuar la oferta informativa a las necesidades de la audiencia. Centrarse en la información útil}

Las ofertas de información generalista no son las más adecuadas para competir en el escenario actual. Uno de los errores de la programación radiofónica espa- 
ñola es la similitud de fórmulas y de temas que llenan las parrillas de las emisoras generalistas, especialmente los contenidos informativos y los espacios de debate y de opinión. Al margen de las duplicidades que existen, muchas de las cuestiones que se abordan no afectan a los intereses inmediatos y cotidianos de los oyentes. Por eso es tan necesaria una mayor presencia de información útil, aquella que facilita a los ciudadanos elementos y recursos válidos para resolver cuestiones que afectan a su vida cotidiana, tanto las referidas al ámbito del ocio como al laboral.

Para conseguir un escenario competitivo, de calidad y adecuado a las necesidades de la audiencia, es necesario modernizar la oferta y ordenar el mapa radiofónico. Pero para eso hace falta un consenso donde la rentabilidad no se mida sólo en concepto de audiencia o de ingresos publicitarios, sino también desde la perspectiva del servicio público que le corresponde a la radio informativa.

\section{Recuperar el espacio de la ficción}

Otro de los retos de la radio es recuperar la ficción, prácticamente desaparecida de las parrillas (Rodero y Soengas, 2010). Los nuevos perfiles de audiencia y los hábitos de consumo más recientes hacen pensar en una buena aceptación de la ficción radiofónica, teniendo en cuenta que en Internet ya triunfan espacios de estas características con unas condiciones de acceso similares a las que se darían en la radio. Pero la ficción radiofónica tiene que adaptarse a los gustos y a los códigos actuales de la audiencia, renovando el lenguaje narrativo y las propuestas. Además, las técnicas de grabación actuales abaratan los costes de la puesta en escena y reducen la complejidad de las producciones, dos de los principales problemas.

La ficción debe distraer, pero también tiene que procurar que los oyentes se vean reflejados en las tramas y que se impliquen emocionalmente en las historias. Siempre se ha dicho que la causa del declive y de la desaparición de la ficción de la radio fue la llegada de la televisión, que conquistó enseguida a la audiencia. Sin despreciar esta teoría, que es cierta en parte, también hay que considerar otras explicaciones alternativas y complementarias que contribuyeron a la desaparición de las radionovelas y del radioteatro: la incorporación de la mujer al mundo laboral y la permanencia de un lenguaje radiofónico demasiado encorsetado y caduco que no evolucionó al mismo ritmo que la sociedad (Balsebre, 1994). La audiencia ya no se sentía identificada en unos relatos anacrónicos desde el punto de vista estético y temático y, en cambio, si se veía reflejada en las propuestas más modernas que aparecían en las series y en los programas de televisión. 


\section{Conclusiones}

La radio tiene que desprenderse de estructuras y de fórmulas obsoletas y proponer alternativas con lenguajes adaptados a los códigos de los nuevos públicos, tanto en el ámbito de la información como en el de la ficción. Una de las prioridades es renovar el concepto de actualidad.

Es necesario un reciclaje de los profesionales para adaptarse a la realidad, algo similar a lo que se hizo durante el proceso de informatización de las redacciones, a finales de los 80 y principios de los 90, aunque en aquella ocasión no todas las emisoras se actualizaron conjuntamente, por falta de recursos o de iniciativa, generando diferencias importantes que se evidenciaban en la calidad de la oferta. No conviene repetir el mismo error para evitar desequilibrios.

Actualmente la radio tiene que competir con la prensa y con la televisión, ya que, gracias a Internet, ofrecen servicios que antes eran propios de las ondas. Los periódicos digitales actualizan sus ediciones cada pocos minutos y los canales de información continua compiten directamente con los boletines horarios y con las emisoras all news. La diferencia ya no puede basarse en la temporalidad, en la actualidad inmediata, en la instantaneidad y en la ubicuidad, características tradicionales y exclusivas de la radio, que le permitían diferenciarse de la prensa y de la televisión. Ahora, gracias a la digitalización, todos los soportes tienes las mismas posibilidades. Entonces, la oferta tiene que basarse en la calidad de los contenidos y en propuestas novedosas y diferentes que atraigan a una audiencia exigente. A la radio le interesa captar al público joven que en estos momentos sintoniza con Internet.

La irrupción de la tecnología en los medios ha generado una evolución en los procesos de producción de la información e incluso en los servicios, algo que requiere una redefinición de los soportes para ajustarse a la realidad. Y en este sentido la radio es uno de los más afectados. La digitalización, la convergencia mediática y la fragmentación, segmentación y especialización de las audiencias determinan de una forma decisiva las exigencias y las necesidades del nuevo escenario. Pero, además, hay que añadir un cuarto factor: la crisis económica, que ha obligado a una reestructuración profunda que afecta a todas las dimensiones del medio.

\section{Referencias}

Balsebre, Armand (1994). El lenguaje radiofónico. Madrid: Cátedra.

Campos Freire, Francisco (coord.) (2011). El nuevo escenario mediático. Zamora: Comunicación Social Ediciones y Publicaciones.

Campos Freire, Francisco (coord.) (2010). El cambio mediático. Zamora: Comunicación Social Ediciones y Publicaciones. 
Cebrián Herreros; Mariano (2001). La radio en la convergencia multimedia. Madrid: Gedisa

Corredoira y Alfonso, Loreto (1999). Los retos de la información en Internet. Las libertades de acceso y difusión, Madrid: UCM.

De Aguinaga, Enrique (2002). El periodista en el umbral del Siglo XXI. En: Estudios sobre el Mensaje Periodístico. Disponible en: http://pendientedemigracion.ucm.es/info/emp/Numer_08/Art/4-01-1.pdf (08/12/2012).

León, Bienvenido (2000). La información audiovisual interactiva en el entorno de convergencia digital: desarrollo y rasgos distintivos. En: Comunicación y sociedad, Vol. 12, $\mathrm{n}^{\mathrm{0}}$ 2. Disponible en: http://www.unav.es/fcom/comunicacionysociedad/es/articulo.php?art_id=126 (17/12/2012)

Lopez Vidales, Nereida (2005). Los medios audiovisuales en el tercer milenio. Atrapados en la tela de araña. En: Telos, $\mathrm{n}^{0}$ 62. Disponible en: http:// sociedadinformacion.fundacion.telefonica.com/telos/articulocuaderno.asp@ idarticulo $=5 \&$ rev $=62 . \mathrm{htm} .(07 / 10 / 2012)$

López Vidales, Nereida y Peñafiel Sainz, Carmen (2000). La tecnología en radio. Principios básicos, desarrollo y revolución digital. Bilbao: Servicio editorial de la Universidad del País Vasco.

Martínez Costa, Pilar (2001) Sobre convergencias y divergencias digitales. Un nuevo paradigma para la radio. En: Sala de prensa, Volumen 2. Disponible en: http://www.saladeprensa.org/art199.htm. (20/102012) Martínez Costa, Pilar (1997). La radio en la era digital. Madrid: El País/Aguilar.

Martínez Soler, José Antonio (1998). Jaque a Polanco. La guerra digital: Un enfrentamiento en las trincheras de la política, el dinero y la prensa. Madrid: Temas de Hoy.

Merayo, Arturo (1996). Tecnología e información en la radio española de fin de siglo. En: Zer, Revista de estudios de comunicación, $\mathrm{n}^{0}$ 1, 193-108. Disponible en: http://www.ehu.es/zer/es/hemeroteca/articulo/tecnologia-e-informacionen-la-radio-espanola-de-fin-de-siglo/7 (15/11/2012).

Ortiz Sobrino, Miguel Angel y López Vidales, Nereida (2011). Radio 3.0 Una nueva radio para una nueva era. La democratización de los contenidos. Madrid: Fragua

Ortiz Sobrino, Miguel Angel y Cuesta Rico, Juan (2003). La radio digital: nuevos perfiles profesionales. Madrid : Instituto Oficial de Radio y Televisión.

Peñafiel Saiz, Carmen (2007). Transformaciones de la radio y la televisión en Europa. Universidad del País Vasco.

Prado, Emili y Franquet Rosa (1998). Convergencia digital en el paraíso tecnológico: claroscuros de una revolución. En: Zer, $n^{0}$ 4. Bilbao: Universidad del País 
Vasco. Disponible en: http://www.ehu.es/zer/hemeroteca/pdfs/zer04-01-prado. pdf. $(9 / 12 / 2012)$.

Reig, Ramón (2011). Los dueños del periodismo. Claves de la estructura mediática mundial y de España. Barcelona: Gedisa.

Rojo Villada, Pedro Antonio (2006). Prensa y convergencia tecnológica: Claves para la definición de un nuevo modelo de negocio periodístico en la Era Digital. En: Zer, mayo. Disponible en: http://www.ehu.es/zer/es/hemeroteca/articulo/ prensa-y-convergencia-tecnologica-claves-para-la-definicion-de-un-nuevo-modelo-de-negocio-periodistico-en-la-era-digital/286 (20-11-2012).

Rodero, Emma y Soengas, Xosé (2010). Ficción radiofónica. Madrid: RTVE

Saiz Olmo, Jesús (1995). La redacción informatizada en radiodifusión sonora. Su incidencia en el proceso de producción de la noticia. El caso de Valencia (Tesis Doctoral). Bellaterra: Universitat Autònoma de Barcelona. Departament de Comunicació Àudio-visual i de Publicitat.

Saiz Olmo, Jesús (1998). Nueva radio para nuevos tiempos con nuevos modos entre nuevos medios. Valencia: Fundación Universitaria San Pablo CEU.

Terceiro, José (1996).La socied@d digit@l.Del homo sapiens al homo digitalis. Madrid: Alianza Editorial.

\section{Referencia de este artículo}

Soengas, Xosé (2013). Retos de la radio en los escenarios de la convergencia digital. En: adComunica. Revista Científica de Estrategias, Tendencias e Innovación en Comunicación, $\mathrm{n}^{\circ} 5$. Castellón: Asociación para el Desarrollo de la Comunicación adComunica, Universidad Complutense de Madrid y Universitat Jaume I, 23-36. DOI: http://dx.doi.org/10.6035/2174-0992.2013.5.3 